\title{
Modeling GeV to very high energy emission from long GRBs
}

\section{Jagdish C. Joshi*}

University of Johannesburg

E-mail: jjagdish@uj.ac.za

\section{Soebur Razzaque}

University of Johannesburg

E-mail: srazzaqueduj.ac.za

Gamma-ray bursts (GRBs) are energetic, transient explosions, which indicate either the death of a massive star $\left(\geq 25 \mathrm{M}_{\odot}\right)$ or the merger of two compact object. In the current understanding, collapsar based GRBs stay active for a long duration $\left(\mathrm{T}_{90}>2 \mathrm{~s}\right)$, and merger based GRBs last for a very short duration $\left(T_{90}<2 \mathrm{~s}\right), \mathrm{T}_{90}$ is the time over which gamma-ray burst emits $90 \%$ of its total measured fluence. A clear understanding of GRB emission can reveal the nature of the central source and the composition of the relativistic jet. Fermi-LAT observations of GRBs have detected a significant power-law component in the energy range $\mathrm{MeV}$ to tens of $\mathrm{GeV}$, in short as well as in long GRBs. The explanation of its origin is still not understood. In this work we have discussed a plausible origin of this component, in the case for long GRBs.

4th Annual Conference on High Energy Astrophysics in Southern Africa

25-27 August, 2016

Cape Town, South Africa

${ }^{*}$ Speaker. 


\section{Introduction}

The emission from a GRB in the early time, for the first tens of seconds, is known as 'Promptemission' while the late emission, which remains active after the prompt emission is known as 'Afterglow emission'. The prompt emission dominates in the gamma-rays (keV-MeV-GeV), while the afterglow emission mainly occurs in X-ray, UV, optical, infrared, radio wavelengths and possibly in gamma-rays. The study of prompt and afterglow emission in GRBs reveals the nature of the central source and the composition of the relativistic jet [1,2]. These explosions can be detected in thermal as well as in non-thermal radiation.

The non-thermal emission from GRBs, which is dominant in MeV energy, can be modelled using the band function [3] and in some cases with an addition of Planck type thermal component $[4,5]$. A hard non-thermal, power-law component appears in GeV energy band [6] in some of the GRBs.

One of the most famous model for explaining the observations of GRBs is the Fireball model. This model was developed by Paczynski [7], Goodman [8], Shemi and Piran [9], Rees and Meszaros [10 - 12]. The radiation dominated outflow from GRBs leads to a relativistic flow due to radiation pressure. In the expansion stage, the physical processes in GRBs results in a mixed composition, (electron, positron, protons and heavy nuclei). In the case of long GRBs the jet composition may also include heavy nuclei which are absent in the case of short gamma-ray bursts.

In this work, we have investigated the case of three long GRBs namely GRB090902B (redshift $z=1.82$ [13]), GRB090926A (redshift $z=2.11$ [14]) and GRB 130427A (redshift $z=0.34$ [6]), in which a power-law component has a photon distribution been observed by Fermi-LAT observations. In case of GRB130427A the power-law has a cutoff- at $70 \mathrm{GeV}$, while for GRB090902B at $11.16 \mathrm{GeV}$ and for GRB090926A it occurs at $1.4 \mathrm{GeV}$. The case of GRB 130427A has also been discussed in [15] using the photo-disintegration model [16-19].

\section{Models of gamma-ray production in GRBs}

The emission of gamma-rays from GRBs can originate from thermal as well as non-thermal processes. In this section we have discussed these processes in detail.

\subsection{Photospheric models}

The injection of huge luminosities from GRBs led to early theoretical investigations of thermal emission, where the emission dominated in hard X-rays up to gamma-rays [20, 21]. The physical scenario can be mapped to the fireball model [20 - 24], where the production of thermal energy in the jet occurs via the dissipation of particle and magnetic energy, between the explosion centre and the photosphere. The photospheric emission [25], has been used to account for the X-ray emission observed in BATSE [26] bursts. In some of the GRBs the prompt [4] and afterglow emission [27] has been modelled by considering the thermalization processes at the source, In particular the photospheric emission efficiency can dominate in the long GRBs [28].

If the central source injects the radiation at a radius $\left(r_{0}\right)$ of $10^{7} \mathrm{~cm}$, with a luminosity $L \sim$ $10^{52} \mathrm{erg} \mathrm{s}^{-1}$, then the temperature of the source, if it is in thermal equilibrium can be calcu- 
lated by the relation $L=4 \pi r_{0}^{2} \sigma T^{4}$, where $\sigma$ is the Stefan-Boltzmann constant with a value $5.67 \times$ $10^{-5} \mathrm{erg} \mathrm{cm}^{-2} \mathrm{~s}^{-1} \mathrm{~K}^{-4}$. The thermal emission for the above parameters will peak at $1.7 \mathrm{MeV}$.

\subsection{Lepto-hadronic models}

The relativistic emission from the GRB fireball leads to the formation of GRB jets. The injected total energy into the GRB jet is distributed into magnetic, leptonic (electrons and positrons) and hadronic (protons and heavy nuclei) components. This distribution of energy leads to a broad, multi-wavelength spectrum that is detected at earth. In general the spectrum at earth, with normalization $A$, maximum flux at break energy $E_{0}$, energy spectrum index before $E_{0}, \alpha$, and after $E_{0}, \beta$, can be fitted empirically by the band function [29],

$$
n(E)=\left\{\begin{array}{lr}
A\left(\frac{E}{100 \mathrm{keV}}\right)^{\alpha} \exp \left(\frac{-\mathrm{E}}{\mathrm{E}_{0}}\right), & (\alpha-\beta) \mathrm{E}_{0} \geq \mathrm{E}, \\
A\left(\frac{(\alpha-\beta) E_{0}}{100 \mathrm{keV}}\right)^{\alpha-\beta} \exp (\beta-\alpha)\left(\frac{\mathrm{E}}{100 \mathrm{keV}}\right)^{\beta}, & (\alpha-\beta) \mathrm{E}_{0}<\mathrm{E}
\end{array}\right.
$$

In general the spectral index distribution varies as, $-1.5<\alpha<0, \beta_{\mathrm{av}} \simeq-2$, while the peak energy can have a range of $\mathrm{keV}$ to $\mathrm{MeV}$ with an average around $\simeq 200 \mathrm{keV}$ [30].The band function in general can be used to model the $\mathrm{MeV}$ non-thermal radiation, which is believed to be produced by the synchrotron emission of relativistic electrons. The explanation of prompt emission due to leptonic synchrotron process has been considered in the literature [30, 31]. In this process the relativistic electrons cool very fast by emitting radiation in the jet magnetic field, so that most of their energy can be channeled to the emitted radiation [32].

Hadronic models for GRBs are mainly used if the observed spectrum from GRBs has more components than the usual band spectrum and the inverse Compton emission cannot explain this deviation at higher energy [33]. In case of GRB 080916C, $100 \mathrm{MeV}-\mathrm{GeV}$ emission can be explained by the proton synchrotron model [34] and the $\mathrm{MeV}$ to $100 \mathrm{GeV}$ radiation from GRB 130427A can be explained by the photo-disintegration of heavy nuclei due to the low energy photons available in the jet of GRB [15].

\section{Photo-disintegration of Heavy nuclei in long GRBs}

In this process, an energetic nuclei, in the frame of GRB jets interact with the isotropic radiation field. During the interaction of the heavy nuclei with the radiation field, the heavy nuclei excites to a higher energy state. The de-excitation produces daughter nuclei, secondary nucleons (protons/neutrons) and gamma-ray photons, as shown in equation (3.1),

$$
A+\gamma \rightarrow A^{*} \rightarrow(A-1)+\gamma+n / p
$$

We follow the same notation for our calculation as discussed in [15]. The notation for the frame of refrences are, (i) The comoving GRB jet frame or wind rest frame is denoted with superscript '", (ii) the lab frame or GRB source frame is denoted with superscript ' $*$ ', (iii) the rest-frame of the nuclei is denoted with superscript ' $"$ ', and (iv) the observer frame with no superscript. The energies in the observer frame, lab frame and comoving jet frame are related by the Lorentz boost factor $\Gamma$ of the bulk GRB outflow and redshift $z$ by the relation $E_{\gamma}=E_{\gamma}^{*} /(1+z)=\Gamma E_{\gamma}^{\prime} /(1+z)$. 
The acceleration of atomic nuclei $(Z)$, in the magnetic field of the GRB can be calculated using [35],

$$
\begin{aligned}
t_{\mathrm{acc}}^{\prime} & =\eta \frac{2 \pi E_{A}^{\prime}}{Z e B^{\prime} c} \\
& \approx 2 \times 10^{-5}\left(\frac{\eta}{10}\right)\left(\frac{Z}{26}\right)^{-1}\left(\frac{B^{\prime}}{27.2 \mathrm{kG}}\right)^{-1}\left(\frac{E_{A}^{\prime}}{\mathrm{TeV}}\right) \mathrm{s}
\end{aligned}
$$

where $E_{A}^{\prime}$ is the energy of the cosmic ray nuclei and $\eta=10$ is a fiducial value for the number of gyroradius required for acceleration. During the acceleration process, due to their charge heavy nuclei will cool down due to synchrotron radiation [35],

$$
\begin{aligned}
t_{\mathrm{syn}}^{\prime} & =\frac{6 \pi m_{p}^{4} c^{3} \Gamma}{\sigma_{T} m_{e}^{2} E_{A}^{\prime} B^{\prime 2}}\left(\frac{A}{Z}\right)^{4} \\
& =5.7 \times 10^{6}\left(\frac{B^{\prime}}{130 \mathrm{kG}}\right)^{-2}\left(\frac{E_{A}^{\prime}}{\mathrm{TeV}}\right)^{-1}\left(\frac{A}{56}\right)^{4}\left(\frac{Z}{26}\right)^{-4} \mathrm{~s}
\end{aligned}
$$

This is too long to be significant in the energy range we are interested. The dynamic timescale of the jet flow at the internal shock, with dissipation radius $R_{\text {in }}$ is calculated using,

$$
t_{\mathrm{dyn}}^{\prime}=\frac{R_{\mathrm{in}}}{\Gamma \mathrm{c}}=0.7\left(\frac{R_{\mathrm{in}}}{2 \times 10^{13} \mathrm{~cm}}\right)\left(\frac{\Gamma}{10^{3}}\right)^{-1} \mathrm{~s}
$$

The interaction of heavy nuclei with low energy photons in the GRB jet can be estimated using the observed differential photon flux at earth, $f_{\gamma}(\varepsilon)$ in units of $\left(\mathrm{MeV}^{-1} \mathrm{~s}^{-1} \mathrm{~cm}^{-2}\right)$ [36],

$$
n_{\gamma}^{\prime}\left(\varepsilon^{\prime}\right)=\frac{2 d_{L}^{2}}{R_{\mathrm{in}}^{2} c} f_{\gamma}\left(\frac{\Gamma \varepsilon^{\prime}}{1+z}\right)
$$

where $R_{\text {in }}$ is the internal shock radius and $d_{L}$ is the luminosity distance to the source and $n_{\gamma}^{\prime}\left(\varepsilon^{\prime}\right)$ is the photon density in the comoving frame. The photo-disintegration time scale of heavy nuclei, calculated using the photon density in the jet frame as defined in 3.5, is given by,

$$
t_{A}^{\prime-1}\left(\gamma_{A}^{\prime}\right)=\frac{c}{2 \gamma_{A}^{\prime 2}} \int_{\varepsilon_{\mathrm{th}}^{\prime \prime}}^{\infty} \varepsilon_{\gamma}^{\prime \prime} \sigma_{A}\left(\varepsilon_{\gamma}^{\prime \prime}\right) d \varepsilon_{\gamma}^{\prime \prime} \int_{\varepsilon_{\gamma}^{\prime \prime} / 2 \gamma_{A}^{\prime}}^{\infty} \frac{n_{\gamma}^{\prime}\left(\varepsilon^{\prime}\right)}{\varepsilon^{\prime 2}} d \varepsilon^{\prime}
$$

Here $\gamma_{A}^{\prime}=E_{A}^{\prime} / m_{A} c^{2}$ is the boost factor of the energetic nuclei, $\varepsilon_{\gamma}^{\prime \prime}=\gamma_{A}^{\prime} \varepsilon^{\prime}\left(1-\beta_{A} \cos \theta\right)$ is the photon energy in the rest frame of the nuclei with an angle $\theta$ between their velocity vectors, $\varepsilon_{\mathrm{th}}^{\prime \prime}$ is the threshold photon energy for the nuclei excitation and $\sigma_{A}\left(\varepsilon_{\gamma}^{\prime \prime}\right)$ is the photo-disintegration crosssection and for a relativistic nuclei with speed $\mathrm{u}, \beta=\frac{u}{c}$. For more details, see [15]. We calculated the time scales for Iron and Oxygen nuclei for GRB130427A, GRB090926A, GRB090902B as shown in figure $1,2,3$ respectively. The $\mathrm{p}-\gamma$ interaction time scale can be calculated using the above formula using a mass number $A=1$ and corresponding interaction cross-section values. All these plots indicate the efficiency of photo-disintegration of heavy nuclei in the jet of these GRBs. 


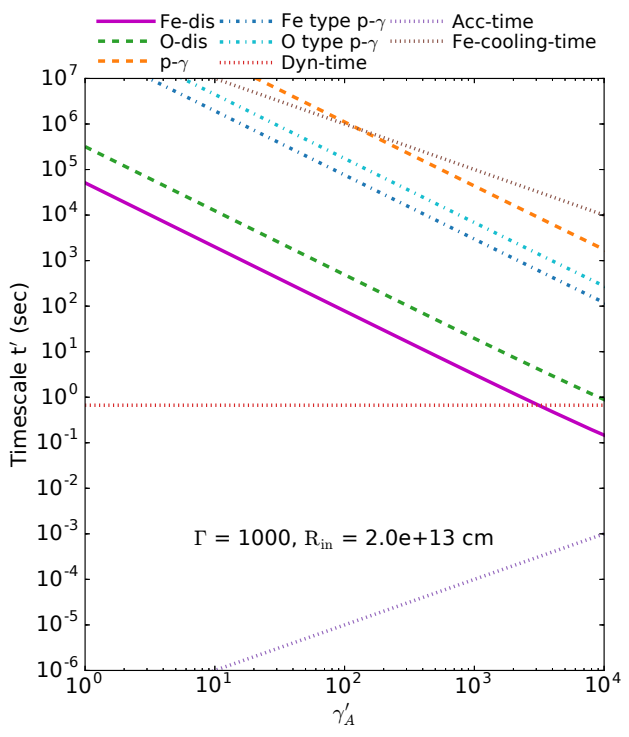

Figure 1: Comparison of different time scales for GRB 130427A.

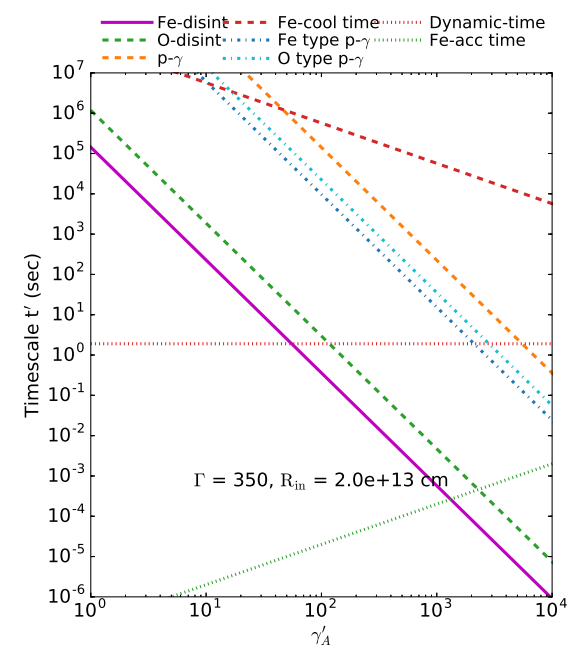

Figure 3: Comparison of different time scales for GRB 090902B.

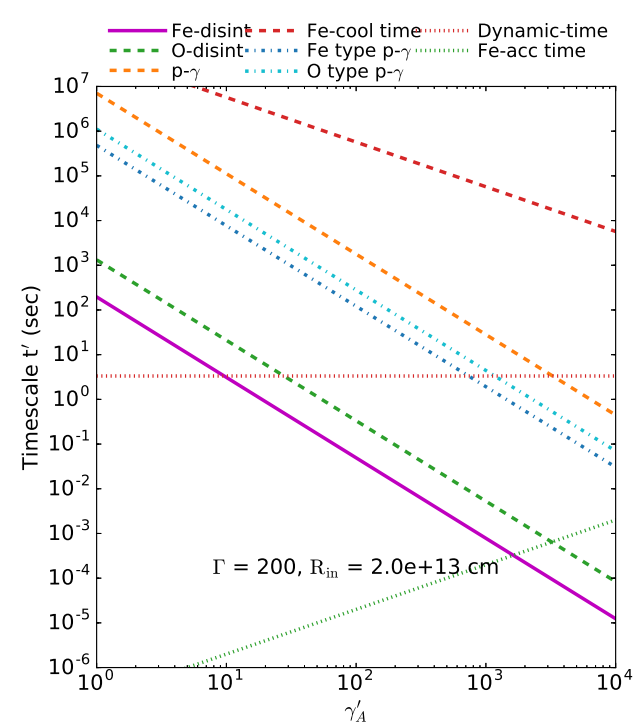

Figure 2: Comparison of different time scales for GRB 090926A.

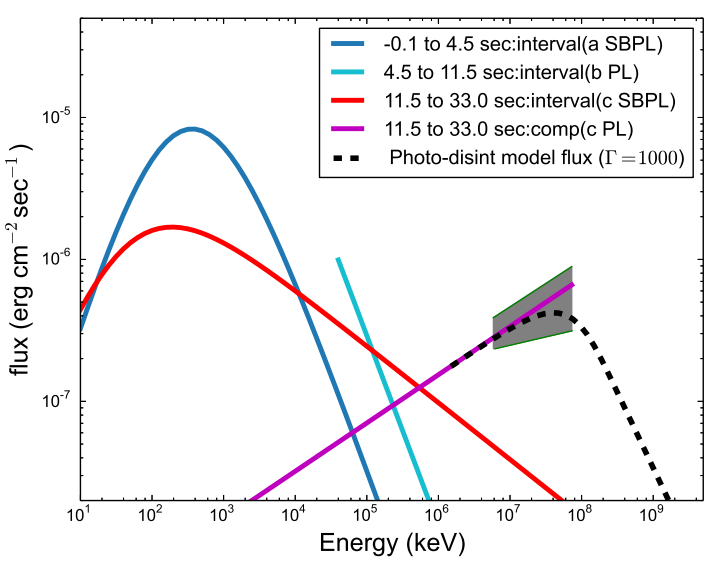

Figure 4: Black dashed line represents the photodisintegrated photons from GRB 130427A.

\section{GeV gamma-ray flux at earth}

The photo-disintegrated gamma-ray flux at earth can be calculated using the formalism given in [15]. In this formalism, heavy nuclei are disintegrated by low energy target photons. The emitted photons have a energy of approximately $1 \mathrm{MeV}$ in the rest frame of nuclei, and further their energy is boosted by the Lorentz factor $\Gamma$ of the GRB jet. These photons will escape the fireball from the optically thin regions. We have followed the same formalism with similar notations. The observed gamma-ray flux at earth for GRB130427A, GRB090926A, GRB090902B are shown in figures 4, 5, 6 respectively, with an effective attenuation due to optical depth effects. 
The Fe- nuclei luminosity to explain these gamma-ray fluxes at earth for GRB 130427A is approximately $10^{53} \mathrm{erg} \mathrm{s}^{-1}$, this is very similar to the luminosity available in the radiation field for this GRB. In the case of GRB090926A and GRB090902B the required luminosity budget is approximately $10^{55} \mathrm{erg} \mathrm{s}^{-1}$, and this is 100 times higher than the radiation field luminosity budget for these GRBs. The luminosity requirement favours the photo-disintegration model for GRB130427A, but other emission mechanism may be in place for the other two GRBs discussed here.

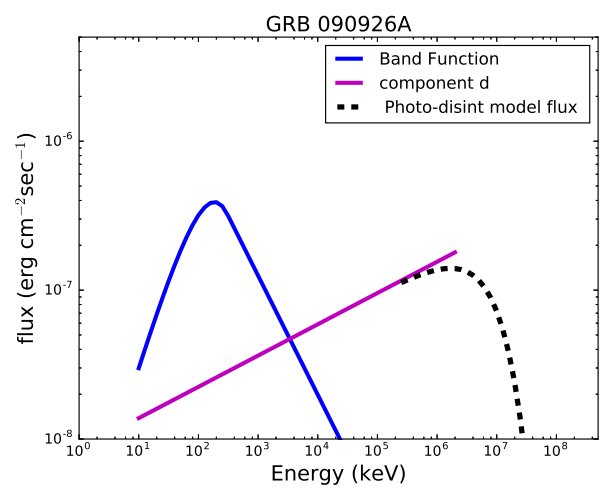

Figure 5: Black dashed line represents the photo-disintegrated photons from GRB 090926A.

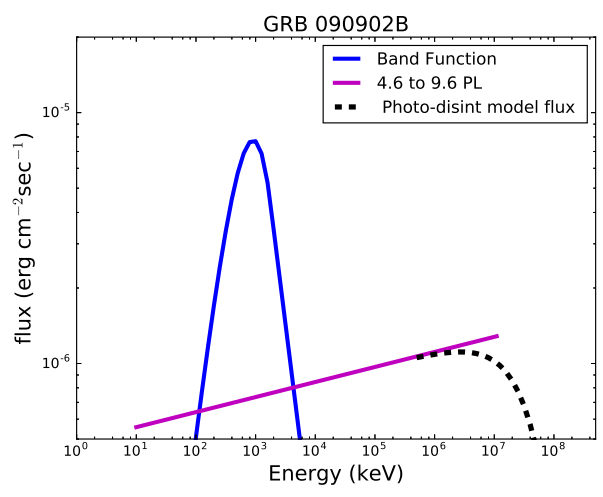

Figure 6: Black dashed line represents the photo-disintegrated photons from GRB 090902B.

\section{Summary and Conclusion}

The observation of GeV gamma-rays from long GRB is very important to understand the dynamics of heavy nuclei composition in GRB jets, if present, in particular because they can produce the power-law component observed in case of long GRBs discussed above. In the case of GRB 130427A, photo-disintegration model can explain the origin of this power-law component with a plausible luminosity budget for this source. We tried the same physical scenario for GRB090926A and GRB090902B and found that only a small portion of the power-law component can be reproduced using photo-disintegration model, but it demands a challenging energy budget.

\section{References}

[1] W.-H. Gao, J. Mao, D. Xu and Y.-Z. Fan, GRB 080916C and GRB 090510: The High-Energy Emission and the Afterglow, ApJL 706 (2009) L33-L36.

[2] Y.-Z. Fan, The spectrum of $\gamma$-ray burst: a clue, MNRAS 403 (2010) 483-490.

[3] D. Band, J. Matteson, L. Ford, B. Schaefer, D. Palmer, B. Teegarden et al., BATSE observations of gamma-ray burst spectra. I - Spectral diversity, ApJ 413 (1993) 281-292.

[4] R. Hascoët, F. Daigne and R. Mochkovitch, Prompt thermal emission in gamma-ray bursts, A\&A 551 (2013) A124.

[5] D. A. Badjin, S. I. Blinnikov and K. A. Postnov, Thermal emission in gamma-ray burst afterglows, MNRAS 432 (2013) 2454-2462. 
[6] M. Ackermann, M. Ajello, K. Asano, W. B. Atwood, M. Axelsson, L. Baldini et al., Fermi-LAT Observations of the Gamma-Ray Burst GRB 130427A, Science 343 (2014) 42-47.

[7] B. Paczynski, Gamma-ray bursters at cosmological distances, ApJL 308 (1986) L43-L46.

[8] J. Goodman, Are gamma-ray bursts optically thick?, ApJL 308 (1986) L47-L50.

[9] A. Shemi and T. Piran, The appearance of cosmic fireballs, ApJL 365 (1990) L55-L58.

[10] P. Meszaros and M. J. Rees, Tidal heating and mass loss in neutron star binaries - Implications for gamma-ray burst models, ApJ 397 (1992) 570-575.

[11] M. J. Rees and P. Meszaros, Relativistic fireballs - Energy conversion and time-scales, MNRAS 258 (1992) 41P-43P.

[12] P. Meszaros and M. J. Rees, High-entropy fireballs and jets in gamma-ray burst sources, MNRAS 257 (1992) 29P-31P.

[13] A. A. Abdo, M. Ackermann, M. Ajello, K. Asano, W. B. Atwood, M. Axelsson et al., Fermi Observations of GRB 090902B: A Distinct Spectral Component in the Prompt and Delayed Emission, ApJL 706 (2009) L138-L144.

[14] M. Ackermann, M. Ajello, K. Asano, M. Axelsson, L. Baldini, J. Ballet et al., Detection of a Spectral Break in the Extra Hard Component of GRB 090926A, ApJ 729 (2011) 114.

[15] J. C. Joshi, S. Razzaque and R. Moharana, Photodisintegrated gamma rays and neutrinos from heavy nuclei in the gamma-ray burst jet of GRB 130427A, MNRAS 458 (2016) L79-L83.

[16] F. W. Stecker, Photodisintegration of Ultrahigh-Energy Cosmic Rays by the Universal Radiation Field, Physical Review 180 (1969) 1264-1266.

[17] J. L. Puget, F. W. Stecker and J. H. Bredekamp, Photonuclear interactions of ultrahigh energy cosmic rays and their astrophysical consequences, ApJ 205 (1976) 638-654.

[18] F. W. Stecker and M. H. Salamon, Photodisintegration of Ultra-High-Energy Cosmic Rays: A New Determination, ApJ 512 (1999) 521-526.

[19] L. A. Anchordoqui, J. F. Beacom, H. Goldberg, S. Palomares-Ruiz and T. J. Weiler, TeV $\gamma$ rays and neutrinos from photodisintegration of nuclei in Cygnus OB2, PRD 75 (2007) 063001.

[20] B. Paczynski, Gamma-ray bursters at cosmological distances, ApJL 308 (1986) L43-L46.

[21] J. Goodman, Are gamma-ray bursts optically thick?, ApJL 308 (1986) L47-L50.

[22] A. Shemi and T. Piran, The appearance of cosmic fireballs, ApJL 365 (1990) L55-L58.

[23] P. Meszaros and M. J. Rees, Tidal heating and mass loss in neutron star binaries - Implications for gamma-ray burst models, ApJ 397 (1992) 570-575.

[24] M. J. Rees and P. Meszaros, Relativistic fireballs - Energy conversion and time-scales, MNRAS 258 (1992) 41P-43P.

[25] P. Mészáros, E. Ramirez-Ruiz, M. J. Rees and B. Zhang, X-Ray-rich Gamma-Ray Bursts, Photospheres, and Variability, ApJ 578 (2002) 812-817.

[26] R. D. Preece, M. S. Briggs, G. N. Pendleton, W. S. Paciesas, J. L. Matteson, D. L. Band et al., BATSE Observations of Gamma-Ray Burst Spectra. III. Low-Energy Behavior of Time-averaged Spectra, ApJ 473 (1996) 310. 
[27] D. A. Badjin, S. I. Blinnikov and K. A. Postnov, Thermal emission in gamma-ray burst afterglows, MNRAS 432 (2013) 2454-2462.

[28] D. Lazzati, B. J. Morsony, R. Margutti and M. C. Begelman, Photospheric Emission as the Dominant Radiation Mechanism in Long-duration Gamma-Ray Bursts, ApJ 765 (2013) 103.

[29] D. Band, J. Matteson, L. Ford, B. Schaefer, D. Palmer, B. Teegarden et al., BATSE observations of gamma-ray burst spectra. I - Spectral diversity, ApJ 413 (1993) 281-292.

[30] A. Pe'er, Physics of Gamma-Ray Bursts Prompt Emission, Advances in Astronomy 2015 (2015) 907321.

[31] H.-F. Yu, H. J. van Eerten, J. Greiner, R. Sari, P. Narayana Bhat, A. von Kienlin et al., The sharpness of gamma-ray burst prompt emission spectra, A\&A 583 (2015) A129.

[32] X.-Y. Wang, Z. Li, Z.-G. Dai and P. Mészáros, GRB 080916C: On the Radiation Origin of the Prompt Emission from keV/MeV TO GeV, ApJL 698 (2009) L98-L102.

[33] K. Asano, S. Guiriec and P. Mészáros, Hadronic Models for the Extra Spectral Component in the Short GRB 090510, ApJL 705 (2009) L191-L194.

[34] S. Razzaque, C. D. Dermer and J. D. Finke, Synchrotron Radiation from Ultra-High Energy Protons and the Fermi Observations of GRB 080916C, The Open Astronomy Journal 3 (2010) 150-155.

[35] X.-Y. Wang, S. Razzaque and P. Mészáros, On the Origin and Survival of Ultra-High-Energy Cosmic-Ray Nuclei in Gamma-Ray Bursts and Hypernovae, ApJ 677 (2008) 432-440.

[36] S. Razzaque, Long-lived PeV-EeV neutrinos from gamma-ray burst blastwave, PRD 88 (2013) 103003. 